\title{
Dielectric Measurements of Poly( $\gamma$-alkyl L-glutamate)s in the Solid State as a Check of Applicability of the Two Phase Model
}

\author{
Naoki SASAKI, Hidehiko Shimodate, * Yuji YAmASHITA,** \\ and Kunio HIKICHI \\ Department of Polymer Science, Faculty of Science, Hokkaido University, \\ Kita-jujo Nish 8-chome, Kita-ku, Sapporo 060, Japan.
}

(Received August 3, 1979)

\begin{abstract}
Molecular motions of the following series of poly( $\gamma$-alkyl L-glutamate)s (PALG)s in the solid state were studied by the method of dielectric measurements; poly( $\gamma$-methyl L-glutamate), poly( $\gamma$-ethyl L-glutamate), poly( $\gamma$-propyl L-glutamate), poly( $\gamma$-butyl L-glutamate), poly( $\gamma$-amyl Lglutamate), and poly ( $\gamma$-hexyl L-glutamate). Each sample exhibits a dispersion near $0^{\circ} \mathrm{C}$ due to sidechain motions. The time-temperature superposition principle was applicable for obtaining the master curve for all samples. The shift factor was well represented by the WLF-equation. The dispersion temperatures of PALG were found to monotonously decrease with increase in the number of carbons in the side-chain alkyl group. Kirkwood's sech-law was applied to obtain the parameter $\bar{\alpha}$, which represents the degree of the breadth of the distribution of the relaxation time. It was found that the distribution of the relaxation time broadens with the increase in the number of carbons in the sidechain alkyl group. These results are discussed in terms of a two phase model in which the rigid $\alpha-$ helical backbone chain is embedded in the flexible side chain.

KEY WORDS Molecular Motion / Poly( $\gamma$-alkyl L-glutamate) / Dielectric Dispersion / Side-Chain Motion / WLF-Equation / Two Phase Model / Kirkwood's Sech-Law /
\end{abstract}

Since the time molecular motions of $\operatorname{poly}(\alpha$-amino acid)s in the solid state were first investigated, ${ }^{1} \mathrm{a}$ considerable body of experimental data has been accour '-lated by the methods of nuclear magnetic resonance, ${ }^{2-4}$ dynamic mechanical, ${ }^{4-8}$ and dielectric measurements. ${ }^{4,9-14}$ According to these results, even in the solid state, long side chains of the $\alpha$ helical poly $(\alpha$-amino acid) undergo considerable motions while the main chain in the $\alpha$-helical structure remains rigid. These results indicate that certain properties of the $\alpha$-helical poly( $\alpha$-amino acid) having the long side chain can be explained if we regard the polymer as a composite system of two independent parts, the side chain and the $\alpha$-helical backbone chain.

The coaxial two phase model consisting of the rigid $\alpha$-helical core and the flexible side chain surrounding it has been proposed to account for the

* Present Address: Emerson \& Cuming Japan K. K., Kuriyama-cho, Yubari-gun, Hokkaido 069-15, Japan.

** Present Address: Hokkai Can Manufacturing Co. Ltd., Iwatsuki 339, Japan. solution properties of poly( $\gamma$-benzyl L-glutamate) $(\mathrm{PBLG})^{15}$ and for the temperature dependence on the X-ray diffraction intensity of lateral reflections for poly ( $\gamma$-methyl L-glutamate) (PMLG) and PBLG in the solid state. ${ }^{16}$ The two phase model had also been adopted to explain the thermal behavior of some $\alpha$-helical poly $(\alpha$-amino acid)s having long side chain. ${ }^{6}$

It may be considered from the view point of this two phase model that the $\alpha$-helical poly $(\alpha$-amino acid) having flexible side chains has a similar structure as the conventional semicrystalline polymer composed of both amorphous and crystalline regions or the amorphous polymer containing the rigid filler. That is, the side chain of $\operatorname{poly}(\alpha$-amino acid) corresponds to the amorphous region of the semicrystalline polymer and the $\alpha$-helical backbone chain to the crystalline region or filler. Thus, the $\alpha$-helical $\operatorname{poly}(\alpha$-amino acid) having a long side chain may behave in a way similar to the semicrystalline polymer with a small degree of crystallinity and the filled amorphous polymer having a small fraction of 
fillers. The increase in the length of the side chain of the $\alpha$-helical poly $(\alpha$-amino acid) may be regarded as a decrease in the degree of crystallinity and in the fraction of the fillers. ${ }^{17}$

In this work, in order to examine the applicability of the two phase model to the $\alpha$-helical poly $(\alpha$-amino acid) in the solid state, dielectric measurements were performed for six poly( $\gamma$-alkyl L-glutamate)s (PALG)s which have a varying number of carbons in the alkyl group at the end of the side chain.

We examined the effects of the variation of the side chain length on the solid state properties of PnALGs in a manner similar to that for examining the semicrystalline polymers, i.e., as a function of the degree of crystallinity.

The polymers used in this work and their abbreviations are given as follows: poly $(\gamma$-methyl L-glutamate) (PMLG), poly( $\gamma$-ethyl L-glutamate) (PELG), $\operatorname{poly}(\gamma$-propyl L-glutamate) (PPLG), poly ( $\gamma$-butyl Lglutamate) (PBtLG), poly( $\gamma$-amyl L-glutamate) (PAmLG), and poly $(\gamma$-hexyl L-glutamate) (PHxLG).

\section{EXPERIMENTAL}

Various PALGs were synthesized by an ester exchange reaction of PMLG(Ajicoat A-2000) with corresponding alcohols. The degree of substitution was checked by proton NMR spectra. For all samples, the exchange reaction was achieved almost completely.

Films of all samples for dielectric measurements were prepared by casting from concentrated chloroform solutions on a glass plate at room temperature. The infrared spectra of films of PALG exhibited amide $\mathrm{V}$ band at about $615-620 \mathrm{~cm}^{-1}$, indicating that in the film, the backbone chain of PALG is in the $\alpha$-helical form.

Silver electrodes were vacuum-deposited on both surfaces of film to assure complete electric contact. Each film was set in the measuring cell and evacuated at a temperature of some $120^{\circ} \mathrm{C}$ until the dielectric constant remained unchanged.

The dielectric constant and loss factor were measured using an Ando Denki TR-1C bridge with a lock -in amplifier LI-573 of NF Circuit Design Block Co., Ltd. and an Ando Denki WBG-5 oscillator. Measurements were made over a frequency range from $30 \mathrm{~Hz}$ to $300 \mathrm{kHz}$ and within a temperature range from -150 to $100^{\circ} \mathrm{C}$.

\section{RESULTS AND DISCUSSION}

Figures 1(a) and (b) show the temperature dependence of $\tan \delta$ measured at a frequency of $1 \mathrm{kHz}$ for PMLG, PELG, and PPLG and for PBtLG, PAmLG, and PHxLG, respectively. Each sample shows two dielectric dispersions, the one located in the vicinity of $0^{\circ} \mathrm{C}$ and the other located at about $-150^{\circ} \mathrm{C}$. For PMLG the dispersion observed in the vicinity of $0^{\circ} \mathrm{C}$ was considered due to the onset of the micro-Brownian motion of the whole side chain, ${ }^{4.6}$ and the dispersion observed at about $-150^{\circ} \mathrm{C}$ was due to the oscillational motion of the side chain on a small scale. ${ }^{4.18 .19}$ Dielectric dispersions observed for other samples will be assigned to the same origins as for PMLG.

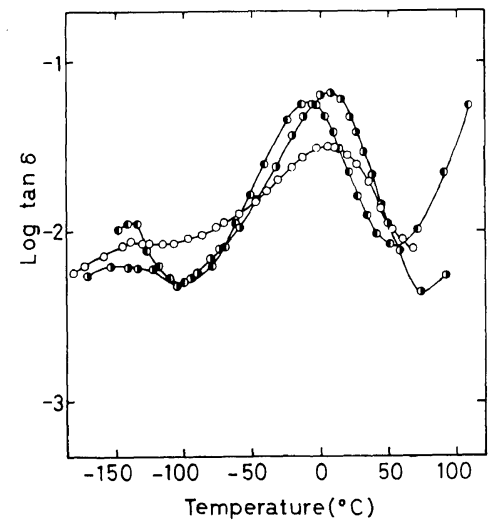

(a)

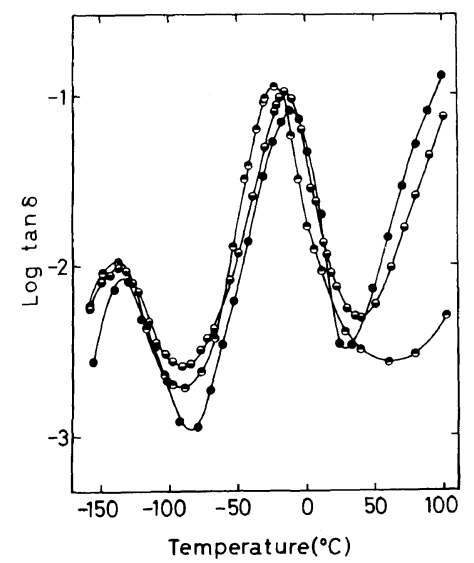

(b)

Figure 1. Temperature dependence of $\tan \delta$ for (a) PMLG (O) PELG (O), and PPLG (O) and (b) PBtLG (๑), PAmLG (Ө), and PHxLG $(\Theta)$ measured at a frequency of $1 \mathrm{kHz}$. 
The temperature region of the dispersion near $0^{\circ} \mathrm{C}$ seemed to shift to lower temperatures on increasing the side-chain length, while that the dispersion at about $-150^{\circ} \mathrm{C}$ remained unchanged for all samples measured. In this paper, we will discuss only the dispersion near $0^{\circ} \mathrm{C}$.

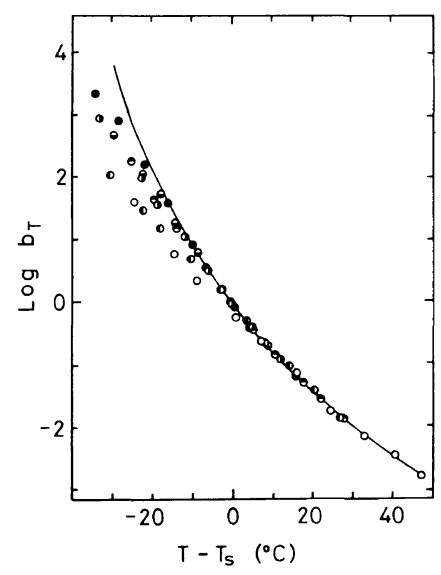

Figure 2. $b_{\mathrm{T}}$ plotted against temperature for PMLG (○), PELG (๑), PPLG (○), PBtLG (๑), PAmLG (๑), and PHxLG $(\Theta)$. Full curve represents the universal WLF-equation.

For all samples measured, the time-temperature superposition principle was found to be applicable to obtain the master curve. Figure 2 shows the shift factor $b_{\mathrm{T}}$, used in the superposition procedure, as a function of temperature. In this figure, the full curve represents the universal WLF-equation ${ }^{20}$

$$
\log b_{\mathrm{T}}=\frac{-8.86\left(T-T_{\mathrm{s}}\right)}{101.6+\left(T-T_{\mathrm{s}}\right)}
$$

For each sample, the temperature dependence of $b_{\mathrm{T}}$ is found to be well expressed by the WLF-equation, if an appropriate choice of the standard temperature $T_{\mathrm{s}}$ is made.

In Figure 3, the temperature dependence of the relaxation strength $\Delta \varepsilon$ is shown. The relaxation strength was determined by the Cole-Cole plot. For all samples, $\Delta \varepsilon$ decreases with increasing temperature.

These behaviors of both $b_{\mathrm{T}}$ and $\Delta \varepsilon$ against temperature indicate that the dispersions observed near $0^{\circ} \mathrm{C}$ for all samples studied have a resemblance to the primary dispersion related to the glass transition of

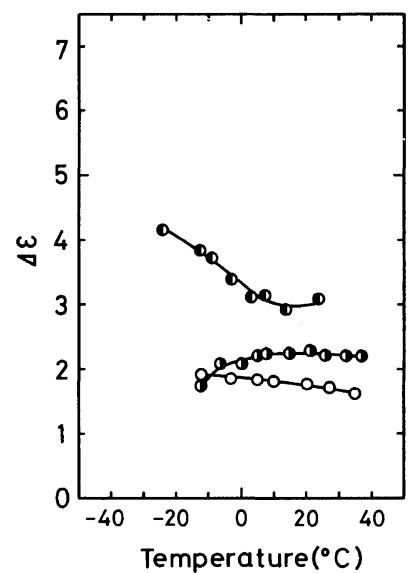

(a)

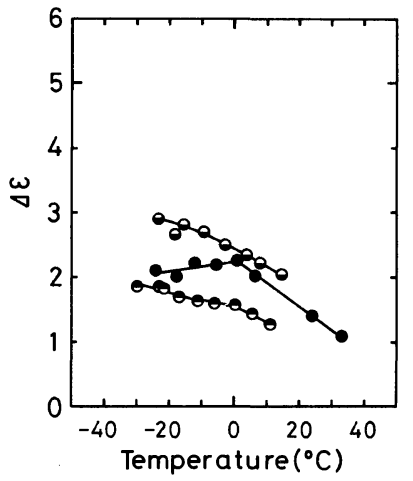

(b)

Figure 3. The relaxation strength plotted against temperature for (a) PMLG (O), PELG (O), and PPLG (O)

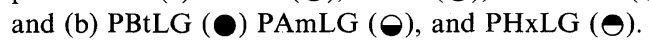

the amorphous polymer, as in the case of PMLG and other poly(glutamate)s. ${ }^{4,6}$

Figure 4 shows the dispersion temperature $T_{\max }$, defined as the temperature of maximum $\tan \delta$, as a function of the number of carbons of the alkyl group in the side chain. As is expected by the glimpse of figure $4, T_{\max }$ is found to decrease with the increase in the side-chain length. This suggests that the sidechain motion is released at lower temperatures as the number of carbons in the alkyl group increases.

The finding that $T_{\max }$ decreases with an increase in the number of carbons of the side-chain alkyl group seems to have good correlation with the observations that $T_{\max }$ of the primary dispersion decreases with a decrease in the degree of crystallinity for the semicrystalline polymers or with the decrease in the 


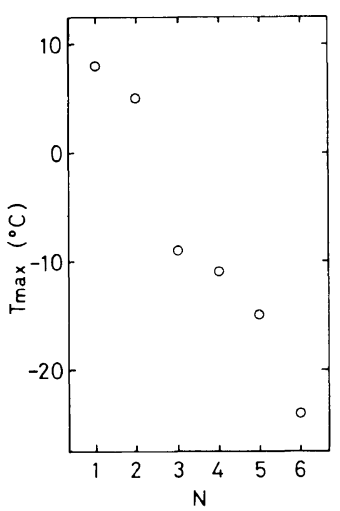

Figure 4. The dispersion temperature $T_{\max }$ as a function of the number of carbons in the side-chain alkyl group.

fraction of fillers for the filled amorphous polymer. ${ }^{21.22 .23}$ The decrease in $T_{\max }$ with an increase in side-chain length suggests a decrease in the interaction between the crystalline region (corresponding to the $\alpha$-helical backbone chain) and the amorphous region (the side chain).

In Figure 5, the master curve of the loss factor for PALGs are compared. Here, the loss factor is normalized with respect to its maximum value and the frequency is reduced with respect to the value at which the maximum loss occurs. Experimental points were omitted for the sake of clarity. The slope at the higher-frequency side of the master curve of the loss factor represents approximately the slope in the wedge-type region of the retardation spectrum except for sign. In the retardation spectrum, the slope of $1 / 2$ has been predicted based on Rouse's Bead-

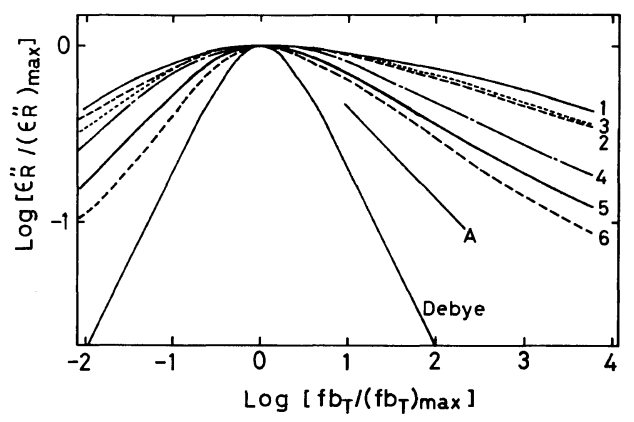

Figure 5. Comparison of master curves of the loss factor. Figures in the diagram represent the number of carbons in the side-chain alkyl group of PALG. The slope of line A corresponds to that theoretically predicted for the amorphous polymer. spring model, which well represents the characteristic features of the relaxation behavior of many amorphous polymers. In this figure, the line A corresponds to the slope of $1 / 2$ in the retardation spectrum.

It has been known that the distribution of the relaxation time of the primary dispersion of the semicrystalline polymer or the filled amorphous polymer is broadened by the increase in the degree of crystallinity or the filler content. ${ }^{22,23}$ It is apparent from Figure 5 that PMLG shows the broadest distribution of the relaxation time, and that the slope on the higher-frequency side of the master curve approaches the slope of $\mathrm{A}$ as the side-chain length increases.

Ishida, et al. examined the primary dielectric dispersion of the semicrystalline polymers as a function of the degree of crystallinity ${ }^{24}$ and discussed their results in the light of Kirkwood's sech-law, ${ }^{25}$

$$
\varepsilon^{\prime \prime} / \varepsilon^{\prime \prime}{ }_{\text {max }}=\operatorname{sech}\left[\bar{\alpha} \cdot \ln \left(f \mid f_{\text {max }}\right)\right]
$$

where $\bar{\alpha}$ is a parameter representing the breadth of the distribution of the relaxation time. $\bar{\alpha}=1$ corresponds to Debye's dispersion; the smaller the value of $\bar{\alpha}$, the broader the master curve. They found that $\bar{\alpha}$ decreases monotonously with an increase in the degree of crystallinity.

Kirkwood's law is simply rewritten as,

$$
\cosh ^{-1}\left(\varepsilon_{\text {max }}^{\prime \prime} / \varepsilon^{\prime \prime}\right)=\bar{\alpha} \ln \left(f \mid f_{\text {max }}\right)
$$

In Figure 6 is shown the left hand side of eq 3 as a function of the logarithm of the reduced frequency in the higher-frequency region. In the region under discussion of the master curve, Kirkwood's sech-law holds well, according to the linear relation between

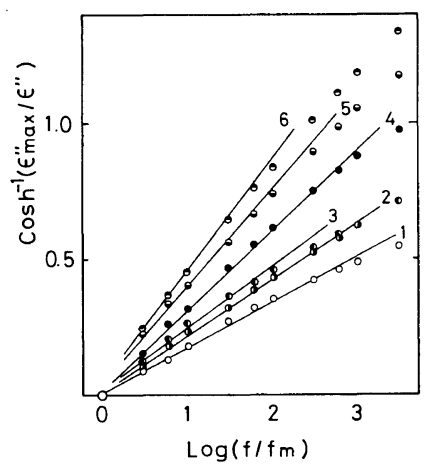

Figure 6. The left hand side of eq $3 \cosh ^{-1}\left[\varepsilon^{\prime \prime}{ }_{\max } / \varepsilon^{\prime \prime}\right]$ plotted against the logarithm of the reduced frequency. 
the both sides of eq 3 . The slope of the plot in the figure gives the value of the parameter, $\bar{\alpha}$.

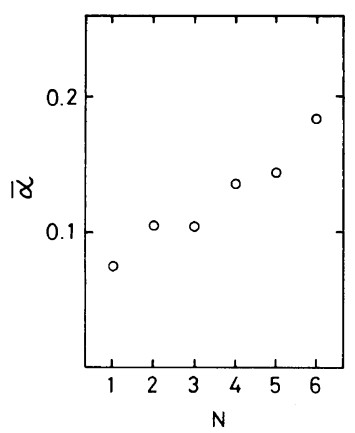

Figure 7. $\bar{\alpha}$ as a function of the number of carbons of the alkyl group in the side chain of PALG.

In Figure 7, the parameter, $\bar{\alpha}$, is plotted against the length of the side chain. It is clearly observed that the value of $\bar{\alpha}$ increases as of the side-chain length increases. These results accord well with those of Ishida's work, if the increase in the side-chain length is regarded as eqivalent to the decrease in the degree of crystallinity.

The fact that variations in $T_{\max }$ and $\bar{\alpha}$ of PALG with the side-chain length have good correlation with $T_{\max }$ and $\bar{\alpha}$ of the semicrystalline polymer and/or the filled amorphous polymer suggests that it is not unreasonable to explain the dielectric behavior at least in terms of the two-phase model, if the change in the side-chain length is assumed to correspond to the variation in the degree of crystallinity or of the fraction of fillers. Certainly the two-phase model is suitable for application to the understanding of the properties of the $\alpha$-helical poly $(\alpha$-amino acid).

Acknowledgment. The authors are indebted to Dr. S. Mori of Ajinomoto Co. for kindly providing poly( $\gamma$-methyl L-glutamate). This work was supported by a Grant-in-Aid for Scientific Research from the Ministry of Education, Culture, and Science of Japan.

\section{REFERENCES}

1. J. A. E. Kail, J. A. Sauer, and A. E. Woodward, $J$. Phys. Chem., 66, 1292 (1963).

2. K. Hikichi, J. Phys. Soc. Jpn., 19, 2169 (1964).

3. A. Tsutsumi, Jpn. J. Appl. Phys., 9, 2225 (1970).

4. A. Tsutsumi, K. Hikichi, T. Takahashi, Y. Yamashita, N. Matsushima, M. Kanke, and M. Kaneko, J. Macromol. Sci.-Phys., B8, 413 (1973).

5. R. G. Saba, J. A. Sauer, and A. E. Woodward, J. Polym. Sci., A, 1, 1843 (1963).

6. Y. Yamashita, A. Tsutsumi, K. Hikichi, and M. Kaneko, Polym. J., 8, 114 (1975).

7. Y. Yamashita, A. Tsutsumi, K. Hikichi, and M. Kaneko, Polym. J., 11, 241 (1979).

8. T. Kajiyama, M. Kuroishi, and M. Takayanagi, $J$. Macromol. Sci.-Phys., B11, 195 (1975).

9. K. Hikichi, K. Saito, M. Kaneko, and J. Furuichi, J. Phys. Soc. Jpn., 19, 557 (1964).

10. S. Makino, K. Kamashima, S. Kubota, and S. Sugai, Jpn. J. Appl. Phys., 3, 55 (1964).

11. S. Sugai, K. Kamashima, S. Makino, and J. Noguchi, J. Polym. Sci., A-2, 4, 183 (1966).

12. A. Tsutsumi, K. Hikichi, M. Mitsumaki, M. Kaneko, and J. Furuichi, J. Phys. Soc. Jpn., 22, 1120 (1967).

13. T. Takahashi, A. Tsutsumi, K. Hikichi, and M. Kaneko, Macromolecules, 7, 806 (1974).

14. N. Sasaki, Y. Yamashita, A. Tsutsumi, and K. Hikichi, Polym. J., 10, 207 (1978).

15. P. J. Flory and W. J. Leonard, Jr., J. Am. Chem. Soc., 87, 2102 (1965).

16. N. Matsushima and K. Hikichi, Polym. J., 10, 437 (1978).

17. Y. Yokomori, Y. Uematsu, and I. Uematsu, Rep. Prog. Polym. Phys. Jpn., 15, 633 (1972).

18. Y. Yamafuji, J. Phys. Soc. Jpn., 15, 2295 (1960).

19. R. Hayakawa and Y. Wada, J. Polym. Sci. Polym. Phys. Ed., 12, 2119 (1974).

20. M. L. Williams, R. F. Landel, and J. D. Ferry, J. Am: Chem. Soc., 77, 3701 (1955).

21. K. H. Illers and H. Breuer, J. Colloid Sci., 18, 1 (1963).

22. R. F. Landel, Trans. Soc. Rheol., 2, 53 (1958).

23. J. D. Ferry, "Viscoelastic Properties of Polymers," John Wiley \& Sons, New York, N.Y., 1960.

24. Y. Ishida, K. Yamafuji, H. Ito, and M. Takayanagi, Kolloid Z. Z. Polym., 184, 97 (1962).

25. R. M. Fuoss and J. G. Kirkwood, J. Am. Chem. Soc., 63, 385 (1941). 